\title{
The von Neumann entropy of networks
}

\author{
Filippo Passerini \\ Perimeter Institute for Theoretical Physics, Waterloo, \\ Ontario N2L 2Y5, Canada and Department of Physics and Astronomy, \\ University of Waterloo, Ontario N2L 3G1, Canada \\ Simone Severini \\ Institute for Quantum Computing and Department of Combinatorics 8 Optimization, \\ University of Waterloo, Waterloo N2L 3G1, ON Canada
}

\begin{abstract}
We normalize the combinatorial Laplacian of a graph by the degree sum, look at its eigenvalues as a probability distribution and then study its Shannon entropy. Equivalently, we represent a graph with a quantum mechanical state and study its von Neumann entropy. At the graph-theoretic level, this quantity may be interpreted as a measure of regularity; it tends to be larger in relation to the number of connected components, long paths and nontrivial symmetries. When the set of vertices is asymptotically large, we prove that regular graphs and the complete graph have equal entropy, and specifically it turns out to be maximum. On the other hand, when the number of edges is fixed, graphs with large cliques appear to minimize the entropy.
\end{abstract}

\section{INTRODUCTION}

The quantum entropy (or, equivalently, von Neumann entropy) was defined by von Neumann around 1927 for proving the irreversibility of quantum measurement processes [28]. Precisely, the quantum entropy is an extension of the Gibbs entropy to the quantum realm and it may be viewed as the average information the experimenter obtains in the repeated observations of many copies of an identically prepared mixed state. It has a fundamental role for studying correlated systems and for defining entanglement measures [29, 30]. In the present work we elaborate on the notion of quantum entropy applied to networks. Since the quantum entropy is defined for quantum states, the first required ingredient is therefore a method to map graphs/networks into states (while the converse is not necessary in our purpose). The literature comprises different ways to associate graphs to certain states or dynamics. Notably, graph-states and spin networks, just to mention two major ones: graph-states are certain quantum error correcting codes, important for characterizing the computational resources in measurement based quantum computation [6, 19]; spin networks are arrangements of interacting quantum mechanical particles, nowadays of great significance for the development of nanotechnologies $3,4,21$. We take a straightforward approach, and take into analysis an entropic quantity for graphs on the basis of a faithful mapping between discrete Laplacians and quantum states, firstly introduced by Braunstein et al. [5] (see also [20]). In synthesis, we see the spectrum of an appropriately normalized Laplacian as a distribution and we compute its Shannon entropy [8] (which measures the amount of uncertainty of a random variable, or the amount of information obtained when its value is revealed). Such a quantity finds a natural place among those global spectral parameters of graphs (i.e., involving the entire spectrum and not just a specific eigenvalue) studied in connection to natural and social networks. For example, the Estrada index, a measure of centrality [15], also used to quantify the degree of folding of long-chain molecules [12, 13, 24]; or the graph energy, that in Hückel theory corresponds to the sum of the energies of all the electrons in a molecule [10, 18]. (See the book chapter [7], for a general review on complexity measures for graphs.) We give evidence that the quantum entropy is a measure of regularity for graphs, i.e., regular graphs have in general higher entropy when the number of edges is fixed. Moreover, entropy seems to depend on the number of connected components, long paths, and nontrivial symmetries. Chosen the number of edges, entropy is smaller for graphs with large cliques and short paths, i.e., graphs in which the vertices form an highly connected cluster. The remainder of the paper is organized as follows. In Section [1 we introduce the required definitions and focus on some basic properties. By adding edges one by one to the empty graph, we try to construct graphs with minimum and maximum entropy. In Section [II] we explore the influence of the graph structure on the entropy. We consider different classes of graphs: regular graphs, random graphs, and the star as an extremal case of scale-free graph (i.e., graphs for which the degree distribution follows a power law). The asymptotic behavior for large number of vertices shows that regular graphs tend to have maximum entropy. We study numerically how the entropy increases when adding edges with different prescriptions. Once fixed the number of edges, the entropy is minimized by graphs with large cliques. Section IV contains remarks and open problems. 


\section{FIRST PROPERTIES}

The state of a quantum mechanical system with a Hilbert space of finite dimension $n$ is described by a density matrix. Each density matrix $\rho$ is a positive semidefinite matrix with $\operatorname{Tr}(\rho)=1$. As we have already mentioned in the introduction, there are many ways to associate graphs to specific density matrices or Hamiltonian evolution (e.g., graph states, bosonic systems, etc.). Here we consider a matrix representation based on the combinatorial Laplacian. Let $G=(V, E)$ be a simple undirected graph with set of vertices $V(G)=\{1,2, \ldots, n\}$ and set of edges $E(G) \subseteq V(G) \times V(G)-\{\{v, v\}: v \in V(G)\}$. The adjacency matrix of $G$ is denoted by $A(G)$ and defined by $[A(G)]_{u, v}=1$ if $\{u, v\} \in E(G)$ and $[A(G)]_{u, v}=0$, otherwise. The degree of a vertex $v \in V(G)$, denoted by $d(v)$, is the number of edges adjacent to $v$. A graph $G$ is $d$-regular if $d(v)=d$ for all $v \in V(G)$. Let $d_{G}$ be the degree-sum of the graph, i.e. $d_{G}=\sum_{v \in V(G)} d(v)$. The average degree of $G$ is defined by $\bar{d}_{G}:=\tilde{n}^{-1} \sum_{v \in V(G)} d(v)$, where $\tilde{n}$ is the number of non-isolated vertices, that is vertices $v$ such that $\{u, v\} \in E(G)$ for some $u \in V(G)$. The degree matrix of $G$ is an $n \times n$ matrix, denoted by $\Delta(G)$, having $u v$-th entry defined as follows: $[\Delta(G)]_{u, v}=d(v)$ if $u=v$ and $[\Delta(G)]_{u, v}=0$, otherwise. The combinatorial Laplacian matrix of a graph $G$ (for short, Laplacian) is the matrix $L(G)=\Delta(G)-A(G)$. The matrix $L(G)$ is a major tool for enumerating spanning trees (via the Matrix-Tree Theorem) and has numerous applications (see Kirchhoff [22], Biggs [2], and Grone et al. [16, 17]). As a consequence of the Geršgorin disc theorem, $L(G)$ is positive semidefinite. By these definitions, the Laplacian of a graph $G$ scaled by the degree-sum of $G$ is a density matrix: $\rho_{G}:=\frac{L(G)}{d_{G}}=\frac{L(G)}{\operatorname{Tr}(\Delta(G))}$. It is then clear that $\rho_{G}=\frac{L(G)}{\tilde{n} \bar{d}_{G}}$. The entropy of a density matrix $\rho$ is defined as $S(\rho)=-\operatorname{Tr}\left(\rho \log _{2} \rho\right)$. Now, given the notion of Laplacian, we say that $S\left(\rho_{G}\right)$ is the quantum entropy (or, for short, entropy) of $G$. Let $\nu_{1} \geq \nu_{2} \geq \cdots \geq \nu_{n}=0$ and $\lambda_{1} \geq \lambda_{2} \geq \cdots \geq \lambda_{n}=0$ be the eigenvalues of $L(G)$ and $\rho_{G}$, respectively. These are related by a scaling factor, i.e. $\lambda_{i}=\frac{\nu_{i}}{d_{G}}=\frac{\nu_{i}}{\tilde{n} \bar{d}_{G}}$, for $i=1, \ldots, n$. The entropy of $\rho_{G}$ can be also written as $S(G)=-\sum_{i=1}^{n} \lambda_{i} \log _{2} \lambda_{i}$, where $0 \log _{2} 0=0$, by convention. (See [27] for a survey on Laplacian spectra.) Since its rows sum up to 0 , then 0 is the smallest eigenvalue of $\rho_{G}$. The number of connected components of $G$ is equal to the multiplicity of 0 as an eigenvalue. The largest Laplacian eigenvalue is bounded by the number of non-isolated vertices, i.e., $\nu_{1} \leq \tilde{n}$ (see Duval et al. [11], Proposition 6.2); thus it follows immediately that $0 \leq \lambda_{i} \leq \frac{1}{d_{G}}$, for $i=1, \ldots, n$. It is important to remark that since $0 \leq \lambda_{i} \leq \frac{1}{d_{G}} \leq 1$, for $i=1, \ldots, n, S\left(\rho_{G}\right)$ equals the Shannon entropy of the probability distribution $\left\{\lambda_{i}\right\}_{i=1}^{n}$. If a general density matrix $\rho$ has an eigenvalue 1 then the other must be 0 and $\rho=\rho^{2}$. In such a case, the density matrix is said to be pure; otherwise, mixed. For later convenience, we define the quantity $R(G):=\frac{1}{n} \sum_{i=1}^{n} \frac{\nu_{i}}{\bar{d}_{G}} \log _{2} \frac{\nu_{i}}{\bar{d}_{G}}$. The disjoint union of graphs $G$ and $H$ is the graph $G^{\prime}=G \uplus H$, whose connected components are $G$ and $H$. We denote by $K_{n}$ the complete graph on $n$ vertices. Let $\mathcal{G}_{n}$ be the set of all graphs on $n$ vertices. The next fact was proved by Braunstein et al. [5]:

Theorem 1 Let $G$ be a graph on $n \geq 2$ vertices. Then $\min _{\mathcal{G}_{n}} S(G)=0$ if and only if $G=K_{2} \biguplus_{j} K_{1}^{(j)}$ and $\max _{\mathcal{G}_{n}} S(G)=\log _{2}(n-1)$ if and only if $G=K_{n}$. When $n=2$, then $\min _{\mathcal{G}_{2}} S(G)=\max _{\mathcal{G}_{n}} S(G)=0$ and $G=K_{2}$.

For general density matrices, $S(\rho)=0$, if $\rho$ is a pure state; $S(\rho)=-\log _{2} \frac{1}{n}=\log _{2} n$ if $\rho=\frac{1}{n} I_{n}$, i.e., a completely random state. The analogue in $\mathcal{G}_{n}$ is $K_{n}$ given that the spectrum or $\rho_{K_{n}}$ is $\left\{\frac{1}{n-1}^{[n-1]}, 0^{[1]}\right\}$. The next result bounds the variation of the entropy under edge addition. Let $G^{\prime}=G+\{x, y\}$, where $V(G)=V\left(G^{\prime}\right)$ and $E\left(G^{\prime}\right)=E(G) \cup\{u, v\}$. An alternative proof could be given by invoking eigenvalues interlacing [10].

Theorem 2 For graphs $G$ and $G^{\prime}=G+\{x, y\}$, we have $S\left(\rho_{G^{\prime}}\right) \geq \frac{d_{G^{\prime}}-2}{d_{G^{\prime}}} S\left(\rho_{G}\right)$.

Proof. Chosen a labeling of $V(G)$, for $G \in \mathcal{G}_{n}$ we can write $A(G)=\sum_{\{u, v\} \in E(G)} A(u, v)$, where $A(u, v)$ is the adjacency matrix of a graph $G(u, v):=\{u, v\} \biguplus_{i=1}^{n-2} K_{1}$. We can then define an $n \times n$ diagonal matrix $\Delta(u, v)$ such that $[\Delta(u, v)]_{u, u}=[\Delta(u, v)]_{v, v}=1$ and $[\Delta(u, v)]_{u, v}=0$ if $u \neq v$. It follows that $\Delta(G)=\sum_{\{u, v\} \in E(G)} \Delta(u, v)$. Then $\rho_{G}=\frac{1}{d_{G}} \sum_{\{u, v\} \in E(G)}(\Delta(u, v)-A(u, v))$. Let $\{|1\rangle,|2\rangle, \ldots,|n\rangle\}$ be the standard basis of $\mathbb{C}^{n}$. By definition, $|i\rangle \equiv\left(0_{1}, 0_{2} \ldots, 0_{i-1}, 1_{i}, 0_{i+1}, \ldots, 0_{n}\right)^{T}$. We associate the pure state $|\{u, v\}\rangle=\frac{1}{\sqrt{2}}(|u\rangle-|v\rangle)$ to the edge $\{u, v\}$. Let $P(u, v)$ be the projector associated to $|\{u, v\}\rangle: P(u, v)=\frac{1}{2}\left(I_{2}-\sigma_{x}\right)$. Then $\rho_{G}=\frac{2}{d_{G}} \sum_{\{u, v\} \in E(G)} P(u, v)$ and $\rho_{G^{\prime}}=\frac{d_{G}}{d_{G^{\prime}}} \rho_{G}+\frac{2}{d_{G^{\prime}}} P(x, y)$. It is well-known that the entropy $S$ is concave (see Ohya and Petz $\left.[30]\right): S\left(\sum_{i=1}^{l} \alpha_{i} \rho_{i}\right) \geq$ $\sum_{i=1}^{l} \alpha_{i} S\left(\rho_{i}\right)$, where $\rho_{i}$ are density matrices and $\alpha_{i} \in \mathbb{R}^{+}$. Hence $S\left(\rho_{G^{\prime}}\right) \geq \frac{d_{G}}{d_{G^{\prime}}} S\left(\rho_{G}\right)+\frac{2}{d_{G^{\prime}}} S(\{x, y\})$. However, since $S(\{x, y\})=0$, the claim is true.

Starting from $K_{2} \biguplus_{j} K_{1}^{(j)}$ (the graph with zero entropy) we can think of a discrete-time process in which we add edges so that the entropy is extremal (resp. maximum or minimum) at every step. Let us denote by $G_{i}^{\max }$ and $G_{i}^{\mathrm{min}}$, $i \geq 1$, the graphs with maximum and minimum entropy at the $i$-th step, respectively. Figure 1 contains $S\left(G_{i}^{\text {max }}\right)$ 
and $S\left(G_{i}^{\min }\right)$ (resp. solid and dashed line) as functions of the number of edges $i=1,2, \ldots, 15$, for graphs in $\mathcal{G}_{6}$. The initial graph is $G_{1}^{\max }=G_{1}^{\min }=K_{2} \biguplus_{j=3}^{6} K_{1}^{(j)}$; the final one is $G_{15}^{\max }=G_{15}^{\min }=K_{6}$. Each edge labeled by $j \leq i$ in the graph $K_{6}$ on the left (resp. right) hand side of Figure 2 is also an edge of $G_{i}^{\max }$ (resp. $G_{i}^{\min }$ ). This illustrates the steps for constructing every $G_{i}^{\max }$ and $G_{i}^{\min }$. It turns out that the vertices of $G_{i}^{\max }$ tend to have "almost equal" or equal degree. In fact $G_{i}^{\max }$ is a $i / 3$-regular graph, for $i=3,6,9,12$. On the other hand, $G_{l(l-1) / 2}^{\min }=K_{l} \biguplus_{j=1}^{6-l} K_{1}^{(j)}$, if $l=3,4,5$. The meaning is without ambiguity: entropy is minimized by those graphs with locally added edges, i.e. edges increasing the number of complete subgraphs (also called cliques). Even if we consider graphs with only six vertices, it is already evident that long paths, nontrivial symmetries and connected components give rise to a larger increase of the entropy. This property is confirmed by further numerical analysis in the next section.

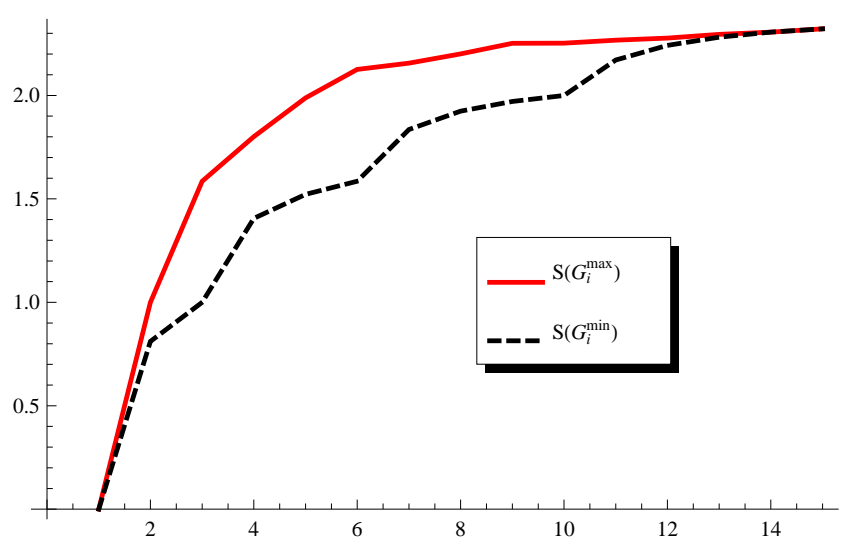

FIG. 1: Plots of $S\left(G_{i}^{\max }\right)$ and $S\left(G_{i}^{\min }\right)$ (resp. solid and dashed line) as functions of the number of edges $i=1,2, \ldots, 15$.
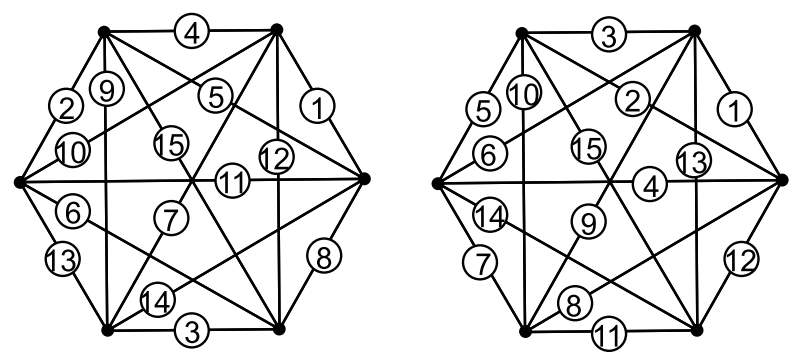

FIG. 2: This figure shows two complete graphs $K_{6}$ with labeled edges. For the graph on the left hand side, the edge labeled by $i$ is added at time $i$ in order to construct $G_{i}^{\max }$. The graph on the right hand side is the analogue drawing for $G_{i}^{\text {min }}$.

\section{ENTROPY AND GRAPH STRUCTURE}

Let $\mathcal{G}_{n, d}$ be the set of all $d$-regular graphs. For $G \in \mathcal{G}_{n, d}$, we have $\Delta(G)=d I_{n}$, and hence $\lambda_{i}=\frac{d-\mu_{i}}{\operatorname{Tr}(\Delta(G))}=\frac{d-\mu_{i}}{d n}$, for $i=1,2, \ldots, n$, where $\mu_{i}$ denotes the $i$-th eigenvalue of $A(G)$.

Theorem 3 Let $G$ be a graph on $n$ nonisolated vertices. If $\lim _{n \rightarrow \infty} \frac{R(G)}{\log _{2} n}=0$ then $\lim _{n \rightarrow \infty} \frac{S(G)}{S\left(K_{n}\right)}=1$. In particular, if $G \in \mathcal{G}_{n, d}$ then $\lim _{n \rightarrow \infty} \frac{S(G)}{S\left(K_{n}\right)}=1$.

Proof. When $G \in \mathcal{G}_{n}, \rho_{G}=\frac{L(G)}{n \bar{d}_{G}}$, where $\bar{d}_{G}=\frac{1}{n} \sum_{v \in V(G)} d(v)$. Since $\lambda_{i}=\frac{\nu_{i}}{n d_{G}}$, we have $S(G)=$ $-\frac{1}{n} \sum_{i=1}^{n} \frac{\nu_{i}}{d_{G}} \log _{2} \frac{\nu_{i}}{d_{G}}+\frac{1}{n} \sum_{i=1}^{n} \frac{\nu_{i}}{d_{G}} \log _{2} n$. Given that $\operatorname{Tr}\left(\rho_{G}\right)=\frac{\nu_{i}}{n d_{G}}=1$, by taking $R(G):=\frac{1}{n} \sum_{i=1}^{n} \frac{\nu_{i}}{d_{G}} \log _{2} \frac{\nu_{i}}{d_{G}}$, the quantum entropy of $G$ is given by $S(G)=-R(G)+\log _{2} n$. Since $S\left(K_{n}\right)=\log _{2}(n-1)$, we have $S(G)=$ 
$-R(G)+\frac{S\left(K_{n}\right) \log _{2} n}{\log _{2}(n-1)}$. From this expression, we see immediately that if $\lim _{n \rightarrow \infty} \frac{R(G)}{\log _{2} n}=0$ then $\lim _{n \rightarrow \infty} \frac{S(G)}{S\left(K_{n}\right)}=1$. Now, let us consider $G \in \mathcal{G}_{n, d}$. Since $d(v)=d$ for every $v \in V(G)$, it follows that $\bar{d}=d$ and $\nu_{i}=d-\mu_{i}$. Given that for a $d$-regular graph $-d \leq \mu_{i} \leq d$, we have $0 \leq \nu_{i} \leq 2 d$, for every $i=1, \ldots, n$. The quantity $R(G)$ is now given by $R(G)=\frac{1}{n} \sum_{i=1}^{n} x_{i} \log _{2} x_{i}$, where $x_{i}=\frac{\nu_{i}}{d}$, and $0 \leq x_{i} \leq 2$. The function $x_{i} \log _{2} x_{i}$ assumes finite values in the range [0,2]. Thus $R(G)$ is also finite. In particular, since $R(G)$ is an average, it remains finite even if considering an arbitrary large number of vertices. This implies that the entropy for a $d$-regular graph tends to the entropy of $K_{n}$ in the limit $n \rightarrow \infty$.

It may useful to remark two points: (1) The simplest regular graph is the perfect matching $M_{n}:=\biguplus_{j=1}^{n / 2} K_{2}^{(j)}$. The density matrix of $M_{n}$ is then $\rho_{M_{n}}=\frac{1}{n} \bigoplus_{n / 2}$ times $\left(\begin{array}{rr}1 & -1 \\ -1 & 1\end{array}\right)$ and $S\left(M_{n}\right)=-\frac{n}{2}\left(\frac{2}{n} \log _{2} \frac{2}{n}\right)=\log _{2} \frac{n}{2}$, because $\lambda_{1}^{\left[\frac{n}{2}\right]}=\frac{n}{2}$ and $\lambda_{2}^{\left[\frac{n}{2}\right]}=0$. Thus, $S\left(M_{n}\right)=S\left(K_{n / 2+1}\right)$. For $M_{4}$ we have $S\left(M_{4}\right)=S\left(K_{3}\right)=1$. More generally, $S\left(M_{2^{k}}\right)=k-1$. (2) The entropy of $G \in \mathcal{G}_{n \rightarrow \infty}$ tends to the entropy of $K_{n}$ if all the quantities $\frac{\nu_{i}}{d_{G}}$ remain finite, i.e., $\lim _{n \rightarrow \infty} \frac{R(G)}{\log _{2} n}=0$.

The complete bipartite graph $K_{p, q}$ has $V\left(K_{p, q}\right)=A \cup B$, where $|A|=p$ and $|B|=q$, and each vertex in $A$ is adjacent to every vertex in $B$. The graph $K_{1, n-1}$ on $n$ vertices is said to be a star.

Theorem 4 Let $G \in \mathcal{G}_{n}$ with $v$ such that $\{v, u\} \in E(G)$ for every $u$, and let $\lim _{n \rightarrow \infty} \bar{d}_{G}=d_{\infty}<\infty$. Then $\lim _{n \rightarrow \infty} \frac{S(G)}{S\left(K_{n}\right)} \leq 1-\frac{1}{d_{\infty}}$. In particular, the star $K_{1, n-1}$ saturates the bound, since $d_{\infty}=2$, and $\lim _{n \rightarrow \infty} S\left(K_{1, n-1}\right) / S\left(K_{n}\right)=\frac{1}{2}$.

Proof. Let $G$ be as in the statement. So, $d_{1}=n-1$. For a graph with at least one edge, Grone et al. (see [17], Corollary 2) proved that $\nu_{1} \geq d_{1}+1$; for a generic graph on $\tilde{n}=n$ vertices, we know that $\nu_{1} \leq n$ (see Duval et al. [11], Proposition 6.2). By these two results, $\nu_{1}=n$. Thus, we have $R(G)=\frac{1}{n} \sum_{i=1}^{n} \frac{\nu_{i}}{d_{G}} \log _{2} \frac{\nu_{i}}{d_{G}}=\frac{1}{d_{G}} \log _{2} \frac{n}{d_{G}}+$ $\frac{1}{n} \sum_{i=2}^{n} \frac{\nu_{i}}{\bar{d}_{G}} \log _{2} \frac{\nu_{i}}{\bar{d}_{G}}$ and $\lim _{n \rightarrow \infty} \frac{R(G)}{S\left(K_{n}\right)} \geq \frac{1}{d_{\infty}}$. Because $S(G)=-R(G)+\log _{2} n$, we have $\lim _{n \rightarrow \infty} \frac{S(G)}{S\left(K_{n}\right)} \leq 1-\frac{1}{d_{\infty}}$. Now, the eigenvalues of $\rho_{K_{1, n-1}}$ are $\lambda_{1}^{[1]}=\frac{n}{2 n-2}, \lambda_{2}^{[n-2]}=\frac{1}{2 n-2}$ and $\lambda_{3}^{[1]}=0$. Thus, the entropy is given by $S\left(K_{1, n-1}\right)=-\frac{n}{2 n-2} \log _{2} \frac{n}{2 n-2}+\frac{n-2}{2 n-2} \log _{2}(2 n-2)$ and in the limit $n \rightarrow \infty$ we have the second part of the statement. Since $\bar{d}=\frac{2 n-2}{n}$, it results $d_{\infty}=2$ and the bound is saturated.

Similarly to what we have done in the previous section, we observe how the entropy of a graph $G \in \mathcal{G}_{n}$ increases as a function of $|E(G)|=e$. Starting from $K_{2} \biguplus_{j=1}^{n-2} K_{1}^{(j)}$, we consider four different ways of adding edges: (i) Random graphs with exactly $e$ edges. These are constructed by chosing $e$ pairs of vertices at random from the total number of pairs; (ii) The graph $M_{2 e} \biguplus_{j=1}^{n-2 e} K_{1}^{(j)}$; (iii) The graph $K_{1,(e+1)-1} \biguplus_{j=1}^{n-e-1} K_{1}^{(j)}$; (iv) The graph $K_{m} \biguplus_{j=1}^{n-m} K_{1}^{(j)}$, where $m=\left[\frac{1+\sqrt{1+8 e}}{2}\right]$. Recall that adding isolated vertices to a graph does not change its entropy. Figure 3 shows the case $n=20$. It is evident that the entropy is larger for graphs with an high number of connected components. In this sense, $M_{n}$ has relatively high entropy. The smallest entropy is obtained for complete graphs.

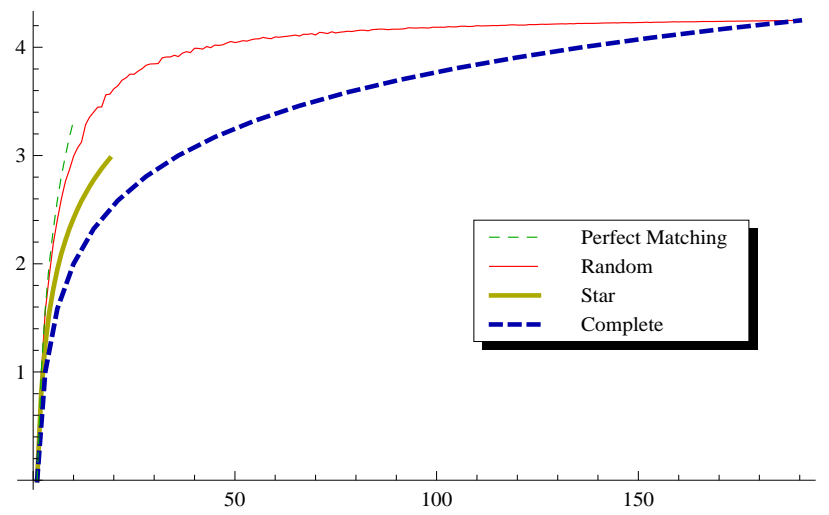

FIG. 3: Plots of the entropy of four different kind of graphs as a function of the number of edges $e=1,2, \ldots, 190$. The different plots represent different ways of adding edges to a graph with $n=20$ vertices. The value of $S\left(R_{n, e}\right)$ has been avaraged over 15 different random graphs, for each value of $e$. 


\section{CONCLUSIONS}

Next is a list of remarks and open problems:

Normalized Laplacian. We have considered the combinatorial laplacian $L(G)$. There is a related matrix called normalized Laplacian and defined by $\mathcal{L}(G)=\Delta^{-1 / 2} L(G) \Delta^{-1 / 2}$ (by convention $\left[\Delta^{-1}\right]_{v, v}=0$ if $d(v)=0$ ). It results that $[\mathcal{L}(G)]_{u, v}=1$ if $u=v$ and $d(v) \neq 0,[\mathcal{L}(G)]_{u, v}=-1 / \sqrt{d(u) d(v)}$ if $\{u, v\} \in E(G)$, and $[\mathcal{L}(G)]_{u, v}=0$, otherwise (see [9, 33]). If a graph has no isolated vertex then $\operatorname{Tr}(\mathcal{L}(G))=n$. Therefore, we can define the density matrix $\widehat{\rho}_{G}:=\frac{\mathcal{L}(G)}{n}$. The entropy of $\widehat{\rho}_{G}$ is then $S\left(\widehat{\rho}_{G}\right)=-\operatorname{Tr}\left(\frac{\mathcal{L}(G)}{n} \log _{2} \frac{\mathcal{L}(G)}{n}\right)=-W+\log _{2} n=-\frac{1}{n} \operatorname{Tr}\left(\mathcal{L}(G) \log _{2} \mathcal{L}(G)\right)+\log _{2} n$. Since the eigenvalues of $\mathcal{L}(G)$ are in $[0,2]$ 9], when limit $n \rightarrow \infty$, the quantity $W$ remains finite. We may then conclude that when the number of vertices goes to infinity, the entropy $S\left(\widehat{\rho}_{G}\right)$ tends to $S\left(\rho_{K_{n}}\right)$. This fact provides a motivation for dealing with $L(G)$ instead of $\mathcal{L}(G)$.

Algebraic connectivity. Let $a(G)=\nu_{n-1}$ be the algebraic connectivity of $G$ [14]. It is nonzero only if $G$ is connected. The value of $a(G)$ quantifies the connectivity of $G$. Is there a relation between $a(G)$ and $S\left(\rho_{G}\right)$ ? Consider $K_{n}$ and the $n$-cycle $C_{n}$, that is the connected 2-regular graph on $n$ vertices. For these, $a\left(K_{n}\right)=n$ and $a\left(C_{n}\right)=2\left(1-\cos \frac{2 \pi}{n}\right)$. By Theorem $3, \lim _{n \rightarrow \infty} S\left(C_{n}\right)=S\left(K_{n}\right)$. However the algebraic connectivity of the two graphs behave differently in this limit: $\lim _{n \rightarrow \infty} a\left(K_{n}\right)=\infty$ and $\lim _{n \rightarrow \infty} a\left(C_{n}\right)=0$.

Eigenvalue gap. Let $b(G)=\mu_{1}-\mu_{2}$ be the eigenvalue gap of $G$. This parameter determines the mixing time of a simple random walk on $G$ (see Lovász [26]). If $G \in \mathcal{G}_{n, d}$ then $a(G)=b(G)$. Hence $\lim _{n \rightarrow \infty} b\left(K_{n}\right)=\infty$ and $\lim _{n \rightarrow \infty} b\left(C_{n}\right)=0$. We can therefore state that $b(G)$ and $S\left(\rho_{G}\right)$ describe different properties of $G$ at least on the basis of this basic observation.

A combinatorial definition. It is unclear whether $S(G)$ is related to combinatorially defined entropic quantities. For example, the Körner entropy defined in [23] (see also Simonyi 32] for a survey) or the entropies defined by Riis [31] and Bianconi [1]. Intuitively, any relation should be weak, because the quantum entropy depends on the eigenvalues. For this reason it describes some global statistical behaviour, with only partial control over combinatorial properties. Beyond cospectrality. Graphs with the same eigenvalues have equal entropy. We have seen that also perfect matchings and complete graphs plus a specific number of isolated vertices have equal entropy, but are clearly noncospectral (see Section [II). Determine families of graphs with the same entropy remains an open problem.

Relative entropy. The quantum relative entropy is a measure of distinguishability between two states (see the review [34]). Given two graphs $G$ and $H$, the quantum relative entropy may be defined as $S(G|| H):=-\operatorname{Tr}\left(\rho_{G} \log _{2} \rho_{H}\right)-$ $S\left(\rho_{G}\right)$. What kind of relations between the two graphs are emphasized by the relative entropy? To what extent can this be used as a measure of distinguishability for graphs?

We conclude with two open problems: does the star $K_{1, n-1}$ have smallest entropy among all connected graphs on $n$ vertices? Is the entropy strictly monotonically increasing under edge addition?

Acknowledgments. The authors would like to thank Michele Arzano, Alioscia Hamma, Dan Lynch, Yasser Omar, Federico Piazza and Samuel Vazquez, for helpful discussion. FP was supported by the Perimeter Institute for Theoretical Physics. Research at Perimeter is supported by the Government of Canada through Industry Canada and by the Province of Ontario through the Ministry of Research \& Innovation. SS was supported by the Institute for Quantum Computing. Research at the Institute for Quantum Computing is supported by DTOARO, ORDCF, CFI, CIFAR, and MITACS.

[1] G. Bianconi, The entropy of network ensembles. arXiv:0802.2888v2 [cond-mat.dis-nn]

[2] N. Biggs, Algebraic Graph Theory, Cambridge, UK: Cambridge University Press, 1993.

[3] B. B. Blinov, D. L. Moehring, L.-M. Duan, and C. Monroe, Nature 428, 153 (2004).

[4] S. Bose, Contemporary Physics, Vol. 48 (1), pp. 13-30, 2007. arXiv:0802.1224v1 [cond-mat.other]

[5] S. Braunstein, S. Ghosh, S. Severini, The laplacian of a graph as a density matrix: a basic combinatorial approach to separability of mixed states, Ann. of Combinatorics, 10, no 3 (2006), 291-317.

[6] H.-J. Briegel and R. Raussendorf, Phys. Rev. Lett. 86, 910 (2001).

[7] D. Bonchev and G. A. Buck, Quantitative Measures of Network Complexity. In: Complexity in Chemistry, Biology and Ecology, D. Bonchev and D. H. Rouvray, Eds., Springer, New York, 2005, p. 191-235.

[8] T. Cover and J. Thomas, Elements of information theory, John Wiley, New York, 1991.

[9] F. R. K. Chung, Spectral graph theory, CBMS Regional Conference Series in Mathematics, 92. Published for the Conference Board of the Mathematical Sciences, Washington, DC; by the American Mathematical Society, Providence, RI, 1997.

[10] D. M. Cvetkovic, M. Doob, H. Sachs, Spectra of graph theory and applications, VEB Deutscher Berlin, Academic Press, New York, 1979. 
[11] A. M. Duval and V. Reiner, Shifted simplicial complexes are Laplacian integral, Trans. Amer. Math. Soc., 354(11):43134344, 2002.

[12] E. Estrada, Characterization of the folding degree of proteins, Bioinformatics 18 (2002), pp. 697-704.

[13] E. Estrada and J. A. Rodríguez-Velázquez, Subgraph centrality in complex networks, Phys. Rev. E 71 (2005), 1-9.

[14] M. Fiedler, Algebraic connectivity of graphs, Czechoslovak Mathematics Journal, 23:298-305, 1973.

[15] L. C. Freeman, A set of measures of centrality based on betweenness, Sociometry, 40, 35-41 (1977).

[16] R. Grone, R. Merris, V. Sunder, The Laplacian spectrum of a graph, SIAM J. Matrix Anal. Appl. 11 (1990), $218-238$.

[17] R. Grone, R. Merris, The Laplacian spectrum of a graph, II. SIAM J. Discrete Math. 7 (1994), no. 2, $221-229$.

[18] I. Gutman, The energy of a graph, Ber. Math. Stat. Sekt. Forschungszentrum Graz., 103: 1-22 (1978).

[19] M. Hein, J. Eisert, and H.J. Briegel. Multi-party entanglement in graph states, Phys. Rev. A 69, 062311 (2004).

[20] R. Hildebrand, S. Mancini, S. Severini, Combinatorial laplacians and positivity under partial transpose, Math. Struct. in Comp. Science (2008), 18, 205-219.

[21] D. Kielpinski, C. Monroe, and D. J. Wineland, Nature 417, 709 (2002).

[22] F. Kirchhoff, Über die Auflösung der Gleichungen, auf welche man bei der Untersuchung der linearen Verteilung galvanischer Ströme gef" uhrt wird. Ann. Phys. Chem. 72 (1847), 497-508.

[23] J. Körner, Coding of an information source having ambiguous alphabet and entropy of graphs, In Proc. 6th Prague Conference on Information Theory (1973), pp. 411-425.

[24] M. Lazić, On the Laplacian energy of a graph, Czechoslovak Math. J. 56(131) (2006), no. 4, $1207-1213$.

[25] H. H. Lieb, M. B. Ruskai, Proof of the strong subadditivity of quantum-mechanical entropy, J. Math. Phys. 14, 1938-1941 (1973).

[26] L. Lovász, Random walks on graphs: a survey. Combinatorics, Paul Erdős is eighty, Vol. 2 (Keszthely, 1993), 353-397, Bolyai Soc. Math. Stud., 2, János Bolyai Math. Soc., Budapest, 1996.

[27] B. Mohar, The Laplacian spectrum of graphs. Graph Theory, Combinatorics, and Applications, 2:871-898, 1991.

[28] J. von Neumann, Mathematische Grundlagen der Quantenmechanik, Berlin, 1932; English translation by R. T. Beyer, Mathematical Foundations of Quantum Mechanics, Princeton, 1955.

[29] M. A. Nielsen and I. L. Chuang, Quantum computation and quantum information, Cambridge University Press, Cambridge, 2000 .

[30] M. Ohya, D. Petz, Quantum entropy and its use. Texts and Monographs in Physics. Springer-Verlag, Berlin, 1993.

[31] S. Riis, Graph Entropy, Network Coding and Guessing games. arXiv:0711.4175v1 [math.CO]

[32] G. Simonyi, Graph entropy. In Combinatorial Optimization, L. L. W. Cook and P. Seymour, Eds., vol. 20 of DIMACS Series on Discrete Math and Computer Science. 1995, pp. 391-441.

[33] A. Terras, Fourier analysis on finite groups and applications, London Mathematical Society Student Texts, 43. Cambridge University Press, Cambridge (1999).

[34] V. Vedral, The role of relative entropy in quantum information theory, Reviews of Modern Physics, vol. 74, Issue 1, pp. 197-234. 